\title{
ON INTEGRAL INEQUALITIES OF GRONWALL-BELLMAN TYPE
}

\author{
EUTIQUIO C. YOUNG
}

\begin{abstract}
A sharp bound is given for solutions of an integral inequality of the Gronwall-Bellman type. The bound which is the exact solution of the corresponding integral equation is obtained by reducing the equation to a system of differential equations.
\end{abstract}

1. Introduction. This paper is concerned with integral inequalities arising from a generalization of the well-known Gronwall-Bellman inequality. For the last decade this inequality has generated a great deal of research activity amongst many mathematicians as it is extended and generalized in various directions. See, for example, the papers of Chandra and Fleishman [3], Chandra and Davis [4], Headley [6], Pachpatte [7], Snow [8], Willet [10], Young [11], the monograph of Walter [9], the lecture notes of Beesack [2] and, more recently, the paper of Abramowich [1]. As pointed out in the papers $[4,5 ; 9$, p. 141, and 12], the sharpest bound for solutions of an integral inequality is provided by the maximal solution or the exact solution of the corresponding integral equation, provided such a solution exists. Thus the results obtained in $[\mathbf{1}, \mathbf{4}, \mathbf{5}, \mathbf{8}, 9$ and 11], which involve Neumann series or Riemann function, are sharp as the bounds are the exact solutions of the corresponding integral equations. In cases where it is not known that a maximal solution or an exact solution of an integral equation exists, an idea of a bound for the solutions of an integral inequality can serve great purpose. Such is the nature, for example, of the results obtained in [7 and $\mathbf{1 0}$ ].

In this paper we shall obtain sharp upper bounds for solutions of the integral inequality

$$
\text { (1) } \begin{aligned}
u(x) \leqslant b(x)+\sum_{j=0}^{n} \int_{0}^{x} a_{0}\left(x_{1}\right) \int_{0}^{x_{1}} a_{1}\left(x_{2}\right) \\
\cdots \int_{0}^{x_{j}} a_{j}\left(x_{j+1}\right) u\left(x_{j+1}\right) \cdots d x_{j+1} \cdots d x_{1},
\end{aligned}
$$

where $x_{0}=x$. When $n=1$ and $b$ is a nonnegative constant, this inequality was considered by Pachpatte [7] for which he gave an upper bound for $u$ which is not sharp.

Received by the editors March 12, 1984. Presented at the Anaheim meeting, January 8-12, 1985 1980 Mathematics Subject Classification. Primary 34A40; Secondary 45A05.

Key nords and phrases. Integral inequalities, maximal, exact solutions, integral equations. 
Although we could as well consider the inequality (1) in $m$-independent variables so that each $x_{j}$ would represent an $m$-vector $x_{j}=\left(x_{j 1}, \ldots, x_{j m}\right)$ and each integral an $m$-fold integral, for convenience we confine our discussion to the case of a single variable. In $\$ 4$ we shall indicate the immediate extension of our result to the $m$-dimensional case.

2. A crude bound. Here we shall obtain a bound for solutions of (1) which is not sharp. This result generalizes Theorem 1 of [7]. Let us define the operator

(2) $T_{k-1} u(x)=\sum_{j=k-1}^{n} \int_{0}^{x} a_{k-1}\left(x_{k}\right) \cdots \int_{0}^{x_{j}} a_{j}\left(x_{j+1}\right) u\left(x_{j+1}\right) d x_{j+1} \cdots d x_{k}$,

with $x_{k-1}=x$ for each $k=1, \ldots, n+1$. It is readily seen that

$$
\left[T_{k-1} u(x)\right]^{\prime}=a_{k-1}(x)\left[u(x)+T_{k} u(x)\right], \quad k=1, \ldots, n+1 .
$$

Further, let us define

$$
v_{k}(x)=v_{k-1}(x)+T_{k-1} u(x), \quad k=1, \ldots, n+1,
$$

with $v_{0}(x) \equiv 0$. It follows that $v_{k-1} \leqslant v_{k}, k=1, \ldots, n+1$, and (1) becomes

$$
u(x) \leqslant b(x)+T_{0} u(x)=b(x)+v_{1}(x) .
$$

THEOREM 1. Let $u, b$ and $a_{j}$ be nonnegative continuous functions for $0 \leqslant x \leqslant T<\infty$, and $a_{j} \not \equiv 0, j=0,1, \ldots, n$. If $u$ satisfies $(1)$, then

$$
u(x) \leqslant b(x)+\int_{0}^{x} a_{0}(s)\left[b(s)+v_{2}(s)\right] d s,
$$

where $v_{2}$ is determined recursively from

$$
v_{k}(x) \leqslant w_{k-2}^{-1}(x) \int_{0}^{x}\left[\left(a_{0}+\cdots+a_{k-1}\right) b+a_{k-1} v_{k+1}\right] w_{k-2}(s) d s,
$$

$k=n, n-1, \ldots, 2$, with

$$
w_{j}(x)=\exp \left(-\int_{0}^{x}\left(a_{0}+\cdots+a_{j}\right) d s\right), \quad j=0,1, \ldots, n,
$$

and

$$
v_{n+1}(x) \leqslant w_{n}^{-1}(x) \int_{0}^{x}\left(a_{0}+\cdots+a_{n}\right) b w_{n} d s .
$$

Proof. From (4) and by (3) and (5) we have

$$
\begin{aligned}
v_{1}^{\prime}(x) & =a_{0}(x)\left[u(x)+T_{1} u(x)\right] \\
& \leqslant a_{0}(x)\left[b(x)+v_{1}(x)+T_{1} u(x)\right] \\
& \leqslant a_{0}(x)\left[b(x)+v_{2}(x)\right],
\end{aligned}
$$

and similarly

$$
\begin{aligned}
v_{2}^{\prime}(x) & =v_{1}^{\prime}(x)+\left[T_{1} u(x)\right]^{\prime} \\
& \leqslant a_{0}(x)\left[b(x)+v_{2}(x)\right]+a_{1}(x)\left[b(x)+v_{3}(x)\right]
\end{aligned}
$$


where $v_{3}(x)=v_{2}(x)+T_{2} u(x)$. By induction we find

$$
\begin{aligned}
v_{k}^{\prime}= & v_{k-1}^{\prime}+\left(T_{k-1} u\right)^{\prime} \\
= & v_{k-1}^{\prime}+a_{k-1}\left(u+T_{k} u\right) \\
\leqslant & \left(a_{0}+\cdots+a_{k-2}\right)\left(b+v_{k}\right) \\
& +a_{k-1}\left(b+v_{k}+T_{k} u\right), \quad k=1, \ldots, n+1,
\end{aligned}
$$

where we have dropped writing the argument of each function, and we define $T_{n+1} u \equiv 0$. Integrating (11) recursively, starting from $k=n+1$ to $k=2$, we obtain (9) and (7). The result (6) then follows from (5) after integrating (10).

When $n=1$, this theorem reduces to Theorem 1 of [7]. As a simple example, consider the case $n=2$ where all the coefficients are positive constants. Then Theorem 1 yields

$$
u \leqslant b\left\{\frac{a_{1}}{a_{0}+a_{1}+a_{2}}+\frac{a_{2} e^{a_{0} x}}{a_{1}+a_{2}}+\frac{\left[a_{0} a_{1} \exp \left(a_{0}+a_{1}+a_{2}\right) x\right]}{\left(a_{1}+a_{2}\right)\left(a_{0}+a_{1}+a_{2}\right)}\right\}
$$

for any nonnegative and continuous function $u$ satisfying the inequality

$$
\begin{aligned}
u(x) \leqslant & b+a_{0} \int_{0}^{x} u d s+a_{0} a_{1} \int_{0}^{x} \int_{0}^{s} u d t d s \\
& +a_{0} a_{1} a_{2} \int_{0}^{x} \int_{0}^{s} \int_{0}^{t} u d r d t d s .
\end{aligned}
$$

The bound in (12) is not sharp as the best bound is provided by the exact solution of the integral equation which is equivalent to the solution of the initial value problem

$$
\begin{gathered}
v^{\prime \prime \prime}-a_{0} v^{\prime \prime}-a_{0} a_{1} v^{\prime}-a_{0} a_{1} a_{2} v=0, \\
v(0)=b, \quad v^{\prime}(0)=a_{0} b, \quad v^{\prime \prime}(0)=a_{0} b\left(a_{0}+a_{1}\right) .
\end{gathered}
$$

3. A sharp bound. We now modify the method used in $\S 2$ and seek a sharp bound for solutions of (1). In essence, our procedure amounts to finding the exact solution of the corresponding integral equation by reducing it to a system of differential equations.

Let $A$ denote the $(n+1) \times(n+1)$ matrix

$$
A(x)=\left[\begin{array}{lllll}
a_{0} & a_{0} & 0 & \cdots & 0 \\
a_{1} & 0 & a_{1} & \cdots & 0 \\
\vdots & \vdots & & & \vdots \\
& & \ddots & & \\
a_{n-1} & 0 & \cdots & 0 & a_{n-1} \\
a_{n} & 0 & \cdots & \cdots & 0
\end{array}\right]
$$

and $B$ the column vector

$$
B=\operatorname{col}\left[\begin{array}{llll}
a_{0} b & a_{1} b & \cdots & a_{n} b
\end{array}\right] .
$$


THEOREM 2. Let $u, b$ and $a_{j}, j=0,1, \ldots, n$ satisfy the same conditions as in Theorem 1. If $u$ satisfies (1), then

$$
u(x) \leqslant b(x)+v_{1}(x), \quad 0 \leqslant x \leqslant T,
$$

where $v_{1}$ is the first component of the $(n+1)$-vector function

$$
V(x)=\int_{0}^{x} Y(x) Y^{-1}(s) B(s) d s
$$

and $Y(x)$ is a fundamental matrix satisfying

$$
Y^{\prime}(x)=A(x) Y(x) .
$$

Proof. With the operator (2) define

$$
v_{k}(x)=T_{k-1} u(x)
$$

and note that $v_{k}(0)=0, k=1, \ldots, n+1$. By (3) we have

$$
\begin{aligned}
v_{k}^{\prime}(x) & =a_{k-1}(x)\left[u(x)+v_{k+1}(x)\right] \\
& \leqslant a_{k-1}(x)\left[b(x)+v_{1}(x)+v_{k+1}(x)\right], \quad k=1, \ldots, n+1,
\end{aligned}
$$

where we have used (5) and defined $v_{n+2} \equiv 0$. Thus treating $v_{1}, \ldots, v_{n+1}$ as components of an $(n+1)$-vector function $V$, the system (19) can be written as

$$
V^{\prime}(x) \leqslant A(x) V(x)+B(x), \quad V(0)=0,
$$

where $A$ and $B$ are defined by (13) and (14), respectively. $V(0)=0$ denotes the zero column vector. As is well knwon, the solution of the intitial value problem $V^{\prime}=A V$ $+B, V(0)=0$, is given by (16).

It is clear that if equality holds in (1), then the same is true in the above derivation so that $w=b+v_{1}$ is the exact solution of the corresponding integral equation.

As a simple example, consider (1) when $n=2, b=a_{0}=a_{1}=1$, and $a_{2}=2$. The system (20) yields

$$
\left[\begin{array}{l}
v_{1} \\
v_{2} \\
v_{3}
\end{array}\right]^{\prime} \leqslant\left[\begin{array}{lll}
1 & 1 & 0 \\
1 & 0 & 1 \\
2 & 0 & 0
\end{array}\right]\left[\begin{array}{l}
v_{1} \\
v_{2} \\
v_{3}
\end{array}\right]+\left[\begin{array}{l}
1 \\
1 \\
2
\end{array}\right]
$$

with $v_{1}(0)=v_{2}(0)=v_{3}(0)=0$ for which a fundamental matrix $Y(x)$ can be readily obtained. We find

$$
\begin{aligned}
v_{1}(x)= & (4 / 7) e^{2 x}+(3 / 7) e^{-x / 2} \cos (\sqrt{3} / 2) x \\
& +(1 / 7 \sqrt{3}) e^{-x / 2} \sin (\sqrt{3} / 2) x-1
\end{aligned}
$$

Hence we have $u \leqslant v_{1}(x)+1=w(x)$. This bound is sharp since $w(x)$ is the solution of the corresponding integral equation which is equivalent to the problem

$$
\begin{gathered}
w^{\prime \prime \prime}-w^{\prime \prime}-w^{\prime}-2 w=0, \\
w(0)=1, \quad w^{\prime}(0)=1, \quad w^{\prime \prime}(0)=2 .
\end{gathered}
$$

4. The $m$-dimensional case. The extension of Theorem 2 to functions of $m$ independent variables $x=x_{0}=\left(x_{01}, \ldots, x_{0 m}\right), m \geqslant 2$, is immediate with obvious interpretation of the integral terms in (1). However, in the conclusion of the theorem, 
the vector function $V$ now satisfies the differential inequality

$$
D_{1} \cdots D_{n} V(x) \leqslant A(x) V(x)+B(x), \quad V(0)=0,
$$

where $D_{i}=\partial / \partial x_{i}, 1 \leqslant i \leqslant m$; or equivalently, $V(x)$ must satisfy the integral inequality

$$
V(x) \leqslant \int_{0}^{x}(A V+B) d s,
$$

where $\int_{0}^{x} d s=\int_{0}^{x_{1}} \cdots \int_{0}^{x_{m}} d s_{1} \cdots d s_{m}$. The exact solution of the integral equation corresponding to (21) can be deduced from Theorem 1 of [4].

\section{REFERENCES}

1. J. Abramowich, On Gronwall and Wendroff type inequalities, Proc. Amer. Math. Soc. 87 (1983), 481-486.

2. P. R. Beesack, Gronwall inequalities, Carleton Univ. Math. Lecture Notes, No. 11, Carleton Univ., Ottawa, Ontario, 1975.

3. J. Chandra and B. A. Fleishman, On a generalization of the Gronwall-Bellman lemma in partially ordered Banach spaces, J. Math. Anal. Appl. 31 (1970), 668-681.

4. J. Chandra and P. Davis, Linear generalization of Gronwall's inequality, Proc. Amer. Math. Soc. 60 (1976), 157-160.

5. S. C. Chu and F. T. Metcalf, On Gronwall's inequality, Proc. Amer. Math. Soc. 18 (1967), 439-440.

6. V. B. Headley, A multidimensional nonlinear Gronwall's inequality, J. Math. Anal. Appl. 47 (1974), $250-255$

7. B. G. Pachpatte, A note on Gronwall-Bellman inequality, J. Math. Anal. Appl. 44 (1973), 758-762.

8. D. R. Snow, Gronwall's inequality for systems of partial differential equations in two independent variables, Proc. Amer. Math. Soc. 33 (1972), 46-54.

9. W. Walter, Differential and integral inequalities, Springer- Verlag, New York, 1970.

10. D. Willet, A linear generalization of Gronwall's inequality, Proc. Amer. Math. Soc. 16 (1965), $774-778$.

11. E. C. Young, Gronwall's inequality in $n$ independent variables, Proc. Amer. Math. Soc. 41 (1973), $241-244$

12. A. D. Ziebur, On the Gronwall-Bellman lemma, J. Math. Anal. Appl. 22 (1968), 92-95.

Department of Mathematics, University of Georgia, Athens, Georgia 30602

Department of Mathematics and Computer Sciences, Florida State University, Tallahassee, FLORIDA 32306 (Current address) 